\title{
ESPÉCIES E FLUTUAÇÃO POPULACIONAL DE CIGARRINHAS EM VIVEIRO DE CITROS, NO MUNICÍPIO DE MOGI-GUAÇU-SP ${ }^{1}$
}

\author{
PEDRO TAKAO YAMAMOTO², SÉRGIO RUFFO ROBERTO ${ }^{3}$, WOLNEY DALLA PRIA JÚNIOR², \\ MARCOS ROGÉRIO FELIPPE ${ }^{4}$, ÉDER PAULO DE FREITAS ${ }^{4}$
}

\begin{abstract}
RESUMO - O presente trabalho teve por objetivo identificar as espécies e flutuação populacional de cigarrinhas em viveiro cítrico localizado em Mogi-Guaçu - SP. O levantamento foi realizado em mudas cítricas da variedade 'Pêra', enxertada sobre limão-'Cravo', iniciando-se em março de $1997 \mathrm{e}$ finalizando-se em março de 1998. Utilizaram-se armadilhas adesivas amarelas, num total de 48 em todo o viveiro e 5 na periferia da mata. A principal espécie capturada foi Bucephalogonia xanthophis. Observaram-se também as espécies Carneocephala sp., Dilobopterus costalimai, Ferrariana trivittata, Lebaja mediana, Macugonalia leucomelas, Plesiommata corniculata e Sonesimia grossa pertencentes à tribo Cicadellini, e Acrogonia sp., Dechacona missionum, Molomea cincta e Oncometopia facialis pertencentes à tribo Proconiini. Durante todo o período de avaliações, foram capturadas cigarrinhas no viveiro, com exceção da primeira quinzena de setembro. As espécies da tribo Cicadellini predominaram em relação às da tribo Proconiini. A tendência da população no outono foi crescente até meados desta estação e, posteriormente, decrescente. No inverno, foi decrescente, na primavera, crescente e, no verão, a população manteve-se estável.
\end{abstract}

Termos de indexação: Citrus sinensis, Proconiini, Cicadellini, armadilha adesiva amarela.

\section{SPECIES AND POPULATION DYNAMICS OF SHARPSHOOTERS IN NURSERY CITRUS, LOCATED AT MOGI-GUAÇU, SP}

\begin{abstract}
This research was an attempt to identify sharpshooter species and their population dynamic in citrus nursery located at Mogi-Guaçú, SP. The survey was performed in nursery trees of 'Pera' sweet orange budded on 'Rangpur' lime, from March 1997 to March 1998. Yellow stick traps were used to collect insects on nursery (48 units) and on adjacent vegetation (5 units). The most prevalent species was Bucephalogonia xanthophis. It was also observed Carneocephala sp., Dilobopterus costalimai, Ferrariana trivittata, Lebaja mediana, Macugonalia leucomelas, Plesiommata corniculata and Sonesimia grossa, which belong to Cicadellini tribe, and Acrogonia sp., Dechacona missionum, Molomea cincta and Oncometopia facialis, belonging to Proconiini tribe. During the whole period of evaluation, the sharpshooters were collected on nursery, except on the first part of September. The Cicadellini species were prevalent in relation to Proconiini ones. There was a tendency of population increase on autumn. On winter the population decreased, on spring increased and on summer the population was stable.
\end{abstract}

Index terms: Citrus sinensis, Proconiini, Cicadellini, yellow stick trap.

\section{INTRODUÇÃO}

No final dos anos 80 , foi relatada, nas regiões Norte e Noroeste do Estado de São Paulo, a Clorose Variegada dos Citros (CVC), que se tornou uma das mais sérias e preocupantes doenças das plantas cítricas (Rossetti et al., 1990). A CVC disseminou-se rapidamente pelas regiões produtoras de citros, sendo hoje encontrada nos Estados de São Paulo, Rio Grande do Sul, Santa Catarina, Paraná, Minas Gerais, Rio de Janeiro, Goiás, Distrito Federal, Bahia, Sergipe e Pará (Rossetti et al., 1997).

O agente causal da CVC é a bactéria limitada ao xilema, Xylella fastidiosa (Chang et al., 1993; Lee et al., 1993; Hartung et al., 1994). Esta bactéria provoca doenças em várias culturas, tais como videira, amendoeira, alfafa, pessegueiro, pereira, cerejeira e outras, sendo transmitida por insetos vetores (Carlos et al., 1997). O patógeno da CVC pode ser transmitido através de borbulhas contaminadas (Jacomino et al., 1993) ou por insetos vetores das famílias Cicadellidae, subfamília Cicadellinae, que se alimentam nos vasos do xilema das plantas (Lopes, 1996; Gravena et al., 1997). Dentre as diversas espécies de cigarrinhas que ocorrem em citros, comprovou-se que Dilobopterus costalimai Young, Acrogonia sp., Oncometopia facialis (Signoret) (Roberto et al., 1996; Lopes et al., 1996), Bucephalogonia xanthophis (Berg), Plesiommata corniculata Young (Krügner et al., 1998), Acrogonia virescens (Metcalf), Ferrariana trivittata (Signoret), Homalodisca ignorata Melichar, Macugonalia leucomelas (Walker), Parathona gratiosa (Blanchard) e Sonesimia grossa

1 (Trabalho 143/2001). Recebido: 12/09/2001. Aceito para publicação: 03/06/2002.

2 Pesquisador Científico, Dr. Centro de Pesquisas Citrícolas - Fundecitrus, Av. Dr. Adhemar Pereira de Barros, 201, C. P. 391, Araraquara, SP. CEP 14801-970. e-mail: ptyamamoto@fundecitrus.com.br

3 Professor Adjunto, Dr. Universidade Estadual de Londrina - UEL. Centro de Ciências Agrárias. Departamento de Agronomia. Caixa Postal 6001. 86051-990. Londrina - PR.

4 Auxiliar de Pesquisa. Centro de Pesquisas Citrícolas - Fundecitrus, Av. Dr. Adhemar Pereira de Barros, 201, C. P. 391, Araraquara, SP. CEP 14801970. 
(Signoret) (Descobertos, 1999) são vetores de $X$. fastidiosa .

Uma das estratégias adotadas para o manejo da CVC é o controle químico dos vetores, inclusive na formação da muda, quando esta se encontra nos viveiros. Nessa condição, Garcia Júnior et al. (1997) realizaram levantamento das cigarrinhas $D$. costalimai, Acrogonia terminalis Young e $O$. facialis, constatando que seus níveis populacionais aumentaram a partir de dezembro, início do verão, permanecendo altos nesta estação e no outono, e diminuindo na primavera e inverno. Em viveiro localizado em Gavião Peixoto, região central do Estado de São Paulo, Roberto et al. (2000) observaram que a espécie B. xanthophis foi a mais abundante. Também foram capturadas as espécies $M$. leucomelas, Dechacona missionum Berg, F. trivittata, Hortensia similis (Walker), Acrogonia sp. e P. corniculata. As cigarrinhas ocorreram durante todo o período de permanência das mudas no campo e foram capturadas tanto na periferia como no centro do viveiro e na vegetação adjacente.

A ocorrência de cigarrinhas em viveiro de mudas cítricas faz com que aumente as chances de contaminação das mudas pela bactéria $X$. fastidiosa. Identificar as espécies, a dinâmica populacional e a abundância durante o processo de produção de mudas é importante para o manejo de cicadelídeos em viveiro e para a consolidação do processo de produção das mudas em ambiente protegido. Visando a identificar as espécies de cicadelídeos de ocorrência em viveiro cítrico e sua flutuação populacional, foi realizado o presente trabalho.

\section{MATERIAL E MÉTODOS}

O trabalho foi realizado em viveiro a céu aberto, localizado no município de Mogi-Guaçu - SP, com irrigação via aspersão. As avaliações iniciaram-se um mês após o plantio dos cavalinhos, da variedade limão- 'Cravo' (Citrus limonia Osbeck) e estendeu-se até a retirada das mudas. O plantio do porta-enxerto foi realizado em fevereiro de 1997, e a enxertia da variedade copa 'Pêra' (Citrus sinensis [L.] Osbeck) foi realizada em julho de 1997, oriunda da borbulheira do Centro de Citricultura Sylvio Moreira/ IAC - Cordeirópolis-SP. O arranquio das mudas deu-se no mês de março de 1998.

A incidência e flutuação populacional de cigarrinhas foram avaliadas por amostragens quinzenais. Utilizaram-se armadilhas adesivas amarelas Stiky Strip (Olson Products Inc., Ohio, EUA), com dimensões de 7,6 x 12,7 cm. Foram distribuídas, uniformemente, 48 armadilhas no viveiro, sendo $28 \mathrm{em}$ toda a periferia do talhão e 20 na área central. As armadilhas foram dispostas em suporte de ferro na altura das plantas e, conforme essas cresciam, as armadilhas também eram remanejadas para estarem na altura máxima das mudas.

Para conhecer a dinâmica populacional de cigarrinhas da mata para o viveiro, foram colocadas 5 armadilhas adesivas amarelas na periferia do viveiro, adjacente à mata, espaçadas a cada 5 metros, penduradas em cerca de arame, a um altura de aproximadamente $1,5 \mathrm{~m}$.

Contou-se o número de cigarrinhas presas à superfície da armadilha, nas duas faces, separadas pela espécie. A freqüência de avaliação foi quinzenal; contudo, a troca das armadilhas foi realizada mensalmente. As avaliações iniciaram-se em março de 1997 e estenderam-se até março de 1998, totalizando
25 avaliações.

Para se determinar os períodos de maior atividade das espécies no viveiro, foram calculados, a partir dos dados mensais de coleta, os valores médios de cigarrinhas capturadas nas diferentes estações do ano: verão (dezembro, janeiro e fevereiro), outono (março, abril e maio), inverno (junho, julho e agosto) e primavera (setembro, outubro e novembro).

A partir dos dados de captura, efetuou-se também a comparação das espécies de cigarrinhas através da porcentagem de ocorrência da espécie nas amostragens mensais, ou seja, pela constância observada a cada ano de avaliação. Em função dos resultados obtidos, as espécies foram distribuídas nas categorias constante, acessória e acidental, de acordo com a classificação de Bodenheimer, citado por Dajoz (1973) e Busoli (1979). A freqüência de cigarrinhas foi estimada pela participação percentual do número de indivíduos dentro da subfamília Cicadellinae.

Durante todo o período de avaliação, realizou-se o controle químico das cigarrinhas em intervalo de aplicação de aproximadamente 7 a 10 dias. Foram realizadas tanto aplicação com pulverizador tratorizado de arrasto como através de pulverização aérea. Os produtos empregados foram escolhidos a critério do Engenheiro Agrônomo responsável pelo viveiro e encontram-se listados na Tabela 1.

Devido à importância de cigarrinhas da subfamília Cicadellinae, que engloba a maioria dos vetores de $X$. fastidiosa nas diversas culturas em que causa doença, analisaram-se somente os dados de espécies desta subfamília de Cicadellidae, e devido ao fato de as espécies da tribo Cicadellini serem consideradas vetores mais eficientes que Proconiini, realizou-se a análise separadamente.

\section{RESULTADOS E DISCUSSÃO}

No período em que foi realizado o levantamento populacional, foram capturadas, na área do viveiro, 14 espécies de cigarrinhas da família Cicadellidae, subfamília Cicadellinae, sendo 10 espécies pertencentes à tribo Cicadellini e 4 espécies à tribo Proconiini (Tabela 2). Dentre as espécies comprovadamente vetoras de X. fastidiosa (Roberto et al., 1996; Lopes et al., 1996; Krügner et al., 1998; Descobertos, 1999), capturaram-se $B$. xanthophis, F. trivittata, M. leucomelas, P. corniculata, Acrogonia sp. e O. facialis.

Considerando-se espécies da subfamília Cicadellinae, as mais freqüentes foram $B$. xanthophis e Acrogonia sp. (Tabela 2 ), sendo respectivamente as mais freqüentes das tribos Cicadellini e Proconiini (Figura 1). A freqüência das espécies $B$. xanthophis e Acrogonia sp., juntas, foi de 69,4\%, enquanto a das demais espécies vetoras de $X$. fastidiosa (D. costalimai, $F$. trivittata, M. leucomelas, $P$. corniculata, $S$. grossa e $O$. facialis), juntas, foi de $19,3 \%$ e a das outras espécies da família Cicadellidae foi de $11,3 \%$ (Tabela 2). Roberto et al. (2000) constataram que $B$. xanthophis foi a espécie mais comum durante todo o processo de formação da muda em viveiro localizado em Gavião PeixotoSP, representando 92,3\% do total de cigarrinhas capturadas.

As espécies B. xanthophis e Acrogonia sp. foram de ocorrência constante no viveiro, $S$. grossa foi de ocorrência acessória e as demais espécies de ocorrência acidental, inclusive as

Rev. Bras. Frutic., Jaboticabal - SP, v. 24, n. 2, p. 389-394, agosto 2002 
espécies $D$. costalimai e $O$. facialis, mais comuns em pomares em produção (Paiva et al., 1998; Roberto \& Yamamoto, 1998). Roberto (1998) constatou que, em laranjeira em produção, as espécies Acrogonia sp., D. costalimai e $O$. facialis, nos anos de 1995 e 1996, foram de ocorrência constante em pomares das regiões Norte, Noroeste e Centro do Estado de São Paulo; entretanto, na região Sul, as espécies Acrogonia sp. e D. costalimai foram de ocorrência acessória e $O$. facialis de ocorrência acidental. Neste mesmo trabalho, o autor constatou, nos anos de 1997 e 1998, que todas as três espécies foram constantes nos pomares das 4 regiões estudadas.

Na periferia da mata capturaram-se 3 espécies da tribo Cicadellini (B. xanthophis, D. costalimai e P. corniculata) e 2 da tribo Proconiini (Acrogonia sp. e O.facialis) (Tabela 2). Todas as espécies capturadas na periferia da mata foram de ocorrência acidental. A freqüência foi de 38,8;27,8; 16,7; 11,1 e 5,6 \%, respectivamente, para D. costalimai, Acrogonia sp., O. facialis, $B$. xanthophis e $P$. corniculata. A espécie $D$. costalimai foi capturada em maior número na periferia da mata que no viveiro, enquanto Acrogonia sp. e B. xanthophis foram capturadas em maior número no viveiro.

As espécies da subfamília Cicadellinae foram capturadas no viveiro, durante todo o período de coleta, com exceção da primeira quinzena de setembro (Figura 2). Ocorreram três picos populacionais, um na primeira quinzena de maio, um outro na primeira quinzena de junho e um terceiro na primeira quinzena de dezembro. Na periferia da mata, ocorreram também três picos populacionais, o primeiro em abril/maio, o segundo em outubro e o terceiro na primeira quinzena de dezembro (Figura 2). No período de junho a outubro, não foram capturados espécimens na periferia da mata.

Comparando-se espécies das tribos Cicadellini e Proconiini, constatou-se que houve predomínio de espécies da primeira tribo (Tabela 2). As espécies da tribo Proconiini predominaram nos primeiros meses de coleta dos dados, da segunda quinzena de abril até o final de maio, e nas demais datas houve predomínio de espécies da tribo Cicadellini.

Em relação às estações do ano, constatou-se que houve um crescimento populacional de cigarrinhas até meados do outono e, posteriormente, uma queda populacional (Figura 3). No inverno, a população foi decrescente, atingindo ao final deste, níveis populacionais muito baixos, enquanto, na primavera, ao contrário, a população foi crescente. No verão, a população de cigarrinhas manteve-se constante, sem tendência de crescimento ou decréscimo.

A baixa população no verão, ao contrário do que foi observado por Garcia Júnior et al. (1997), provavelmente, seja decorrente do estágio das mudas. Nesta estação, as mudas encontravam-se na fase final de produção e, provavelmente, pouco atrativa para as cigarrinhas devido à pouca vegetação e maior porte das mudas.

Apesar do controle químico sistemático realizado no viveiro, mesmo com pulverizações aéreas, constatou-se a presença de cigarrinhas em praticamente todo o processo de formação da muda. Roberto et al. (2000) constataram a presença de cigarrinhas durante todo o processo de formação da muda em

TABELA 1 - Aplicação de inseticidas no viveiro para controle de cigarrinhas (cicadellidae), Mogi-Guaçu - SP.

\begin{tabular}{|c|c|c|c|}
\hline D ata & Inseticida & $\begin{array}{c}\text { Dose } \\
(\mathrm{ml} \text { ou g PC/100 L ou ha })\end{array}$ & $\begin{array}{l}\text { Modalidade de } \\
\text { aplicação }\end{array}$ \\
\hline $13-3-97$ & Fenpropathrin & 50 & Tratorizada \\
\hline $26-3$ & Carbosulfan + óleovegetal + abamectin & $500+1500+100$ & A érea \\
\hline $4-4$ & Carbosulfan + óleovegetal & $75+250$ & Tratorizada \\
\hline $9-4$ & Carbaril & 200 & Tratorizada \\
\hline $14-4$ & Im idacloprid & 5 & Tratorizada \\
\hline $24-4$ & Fenpropathrin + óleovegetal + abamectin & $300+1500+75$ & A érea \\
\hline $2-5$ & A bamectin & 9 & Tratorizada \\
\hline $15-5$ & Im idacloprid & 5 & Tratorizada \\
\hline $4-6$ & Abamectin & 9 & Tratorizada \\
\hline $23-6$ & Fenpropathrin & 300 & A érea \\
\hline $4-7$ & Deltamethrin & 15 & Tratorizada \\
\hline $18-7$ & Metidathion & 1000 & A érea \\
\hline $1-8$ & Clorpiriphos & 1500 & A érea \\
\hline $8-8$ & Fenpropathrin + metidathion & $50+100$ & Tratorizada \\
\hline $22-8$ & Fenpropathrin & 300 & A érea \\
\hline $5-9$ & Im idacloprid & 30 & A érea \\
\hline $19-9$ & Carbaril & 200 & Tratorizada \\
\hline $1^{\circ}-10$ & Im idacloprid & 5 & Tratorizada \\
\hline $16-10$ & Matidathin + óleovegetal + abamectin & $1000+1500+75$ & A érea \\
\hline $30-10$ & Deltame thrin & 500 & A érea \\
\hline $11-11$ & Deltamethrin & 500 & A érea \\
\hline $28-11$ & Im idacloprid & 30 & A érea \\
\hline $8-12$ & Clorpiriphos & 1,5 & A érea \\
\hline $11-12$ & Fenpropathrin & 300 & A érea \\
\hline $19-12$ & Deltame thrin & 500 & A érea \\
\hline $13-1-98$ & Fenpropathrin & 300 & A érea \\
\hline $21-1$ & Abamectin + óleovegetal & $8+250$ & Tratorizada \\
\hline $23-1$ & Metidathion & 1000 & A érea \\
\hline $9-2$ & Fenpropathrin & 300 & A érea \\
\hline
\end{tabular}


TABELA 2 - Número total de insetos, constância e freqüência de cigarrinhas (Hemiptera: Cicadellidae) coletadas através de armadilha adesiva amarela em viveiro cítrico e na periferia da mata em Mogi-Guaçu-SP.

\begin{tabular}{|c|c|c|c|c|c|c|}
\hline \multirow[b]{2}{*}{ Espécies } & \multicolumn{3}{|c|}{ Viveiro } & \multicolumn{3}{|c|}{ Periferia da mata } \\
\hline & $\begin{array}{l}\text { Total de } \\
\text { insetos }\end{array}$ & Constância & $\begin{array}{c}\text { Freqüência } \\
(\%)\end{array}$ & $\begin{array}{l}\text { Total de } \\
\text { insetos }\end{array}$ & Constância & $\begin{array}{c}\text { Freqüência } \\
(\%)\end{array}$ \\
\hline \multicolumn{7}{|l|}{ Cicadellini } \\
\hline B. xanthophis & 64 & Constante & 42,7 & 2 & Acidental & 11,1 \\
\hline Carneocephala sp. & 3 & Acidental & 2,0 & 0 & $--^{1 /}$ & -- \\
\hline D. costalimai & 2 & Acidental & 1,3 & 7 & Acidental & 38,8 \\
\hline F. trivittata & 8 & Acidental & 2,0 & 0 & -- & -- \\
\hline H. similis & 2 & Acidental & 5,3 & 0 & -- & -- \\
\hline L. mediana & 3 & Acidental & 1,3 & 0 & -- & -- \\
\hline M. leucomelas & 3 & Acidental & 2,0 & 0 & -- & -- \\
\hline P. corniculata & 7 & Acidental & 4,7 & 1 & Acidental & 5,6 \\
\hline S. grossa & 6 & Acessória & 4,0 & 0 & -- & -- \\
\hline Outra & 1 & Acidental & 0,7 & 0 & -- & -- \\
\hline \multicolumn{7}{|l|}{ Proconiini } \\
\hline Acrogonia sp. & 40 & Constante & 26,7 & 5 & Acidental & 27,8 \\
\hline D. missionum & 3 & Acidental & 2,0 & 0 & -- & -- \\
\hline M. cincta & 5 & Acidental & 3,0 & 0 & -- & -- \\
\hline O. facialis & 3 & Acidental & 2,0 & 3 & Acidental & 16,7 \\
\hline Total & 150 & - & 100 & 18 & - & 100 \\
\hline
\end{tabular}

1/ A espécie não foi capturada na periferia da mata.
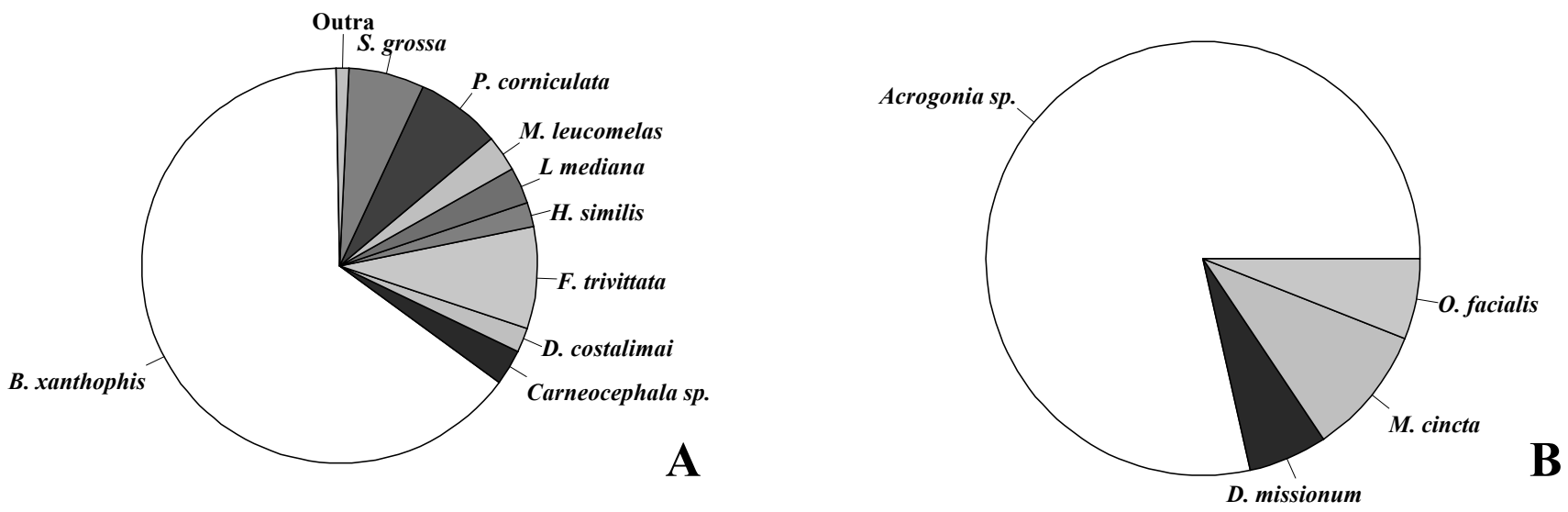

FIGURA 1 - Distribuição de espécies de cigarrinhas das tribos Cicadellini (A) e Proconiini (B) em viveiro de mudas cítricas. 


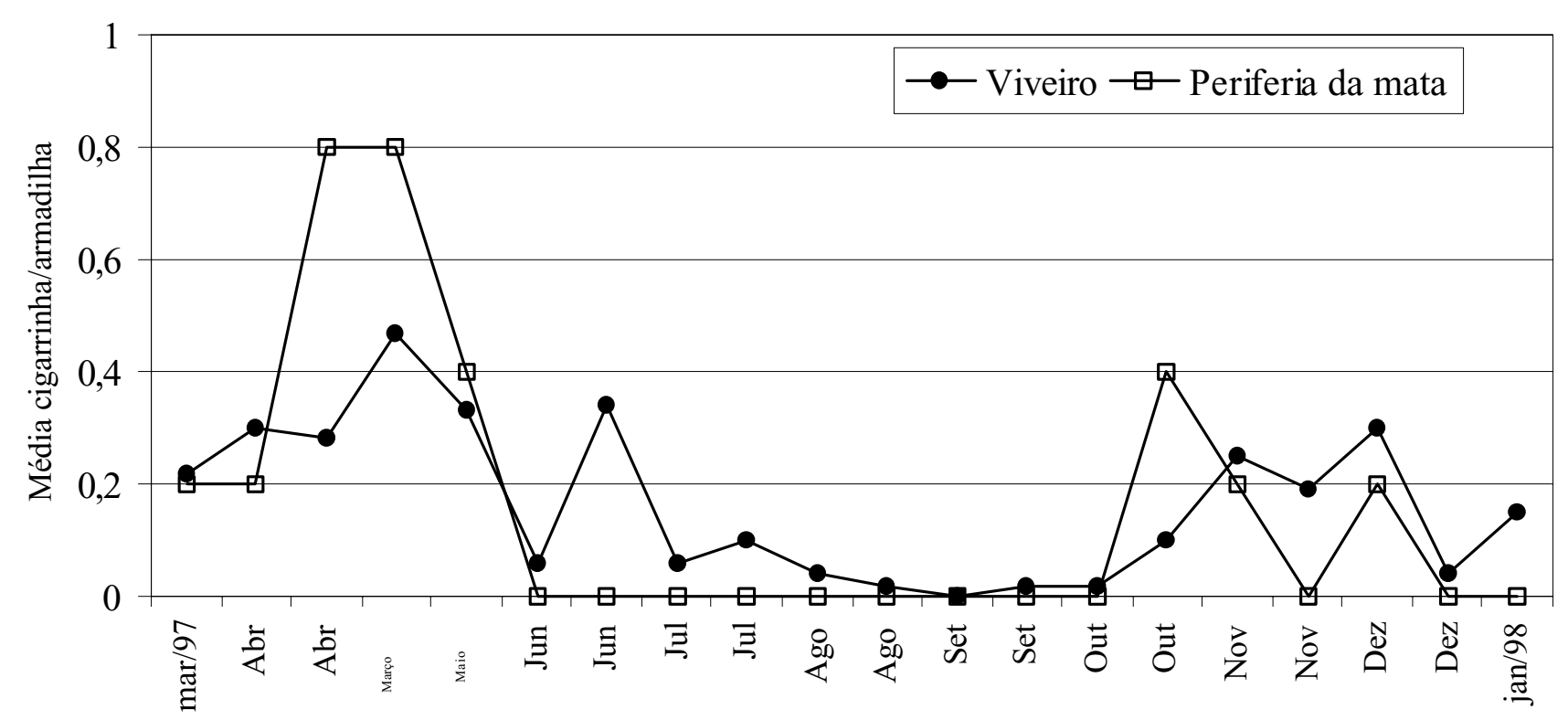

Meses

FIGURA 2 - Flutuação populacional de cigarrinhas (Hemiptera: Cicadellidae) em mudas cítricas e na periferia de mata, adjacente ao viveiro.
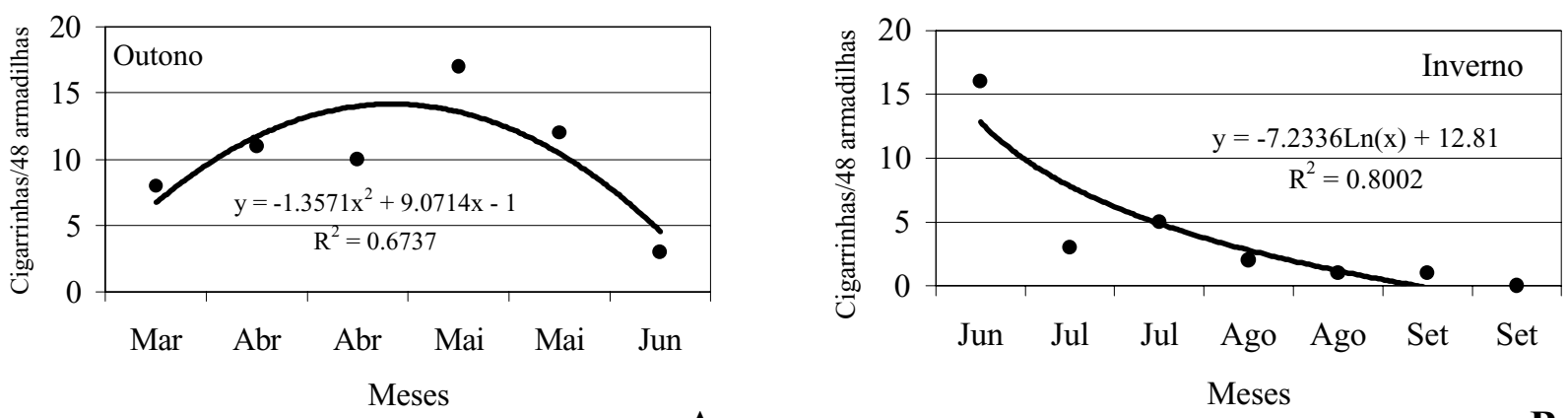

A

B
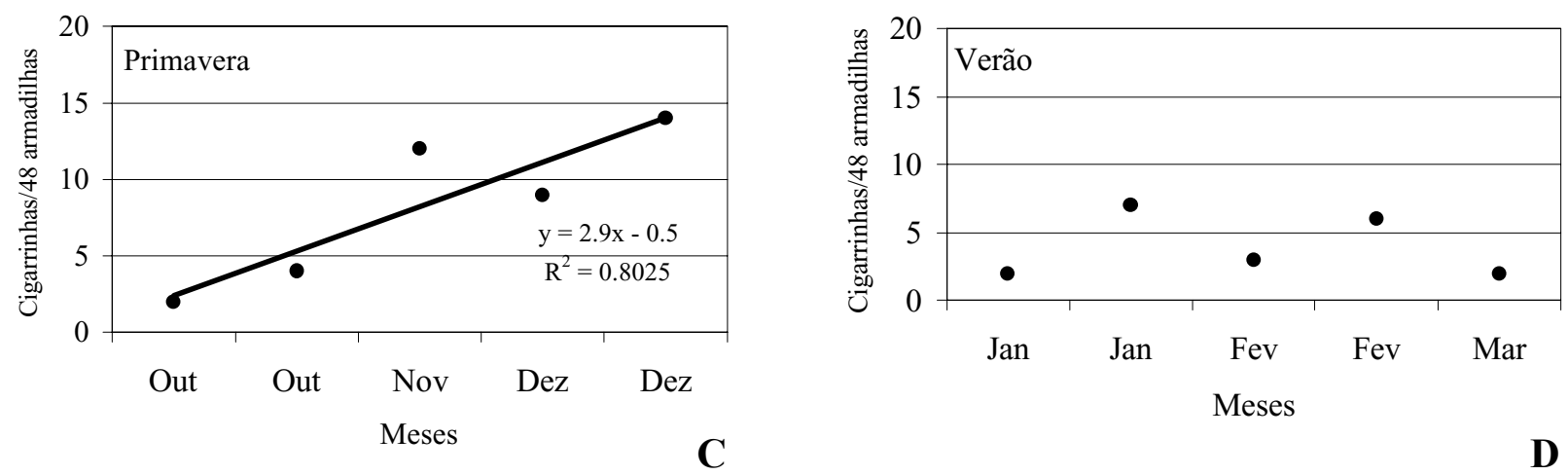

C

D

FIGURA 3 - Flutuação populacional de cigarrinhas em viveiro de mudas cítricas no período de março de 1997 a março de 1998 , no outono (A), inverno (B), primavera (C) e verão (D). 
viveiro localizado em Gavião Peixoto-SP. A ocorrência de cigarrinhas em viveiro pode levar à contaminação das mudas pela bactéria $X$. fastidiosa, causadora da CVC. Entretanto, a contaminação estará relacionada à infectividade das cigarrinhas vetoras da bactéria.

\section{CONCLUSÕES}

1. Em viveiro cítrico há predomínio da espécie B. xanthophis.

2. Durante todo o processo de formação de mudas, ocorre presença de cigarrinhas no viveiro, com predomínio de espécies da tribo Cicadellini.

3. A população de cigarrinhas é crescente até meados do outono e, posteriormente, decrescente.

4. No inverno, a população é decrescente e, na primavera, crescente.

5. No verão, a população de cigarrinhas não apresenta um padrão de crescimento ou decréscimo.

\section{REFERÊNCIAS BIBLIOGRÁFICAS}

BUSOLI, A.C. Levantamento, constância e flutuação populacional de alguns insetos coletados com armadilhas luminosas em Jaboticabal - SP. 1979. 100f. Dissertação (Mestrado em Entomologia), Escola Superior de Agricultura Luiz de Queiroz, Universidade de São Paulo, Piraciacaba, 1979.

CARLOS, E.F.; RODRIGUES NETO, J.; BERETTA, M.J.G. A bactéria Xylella fastidiosa. In: DONADIO, L. C.; MOREIRA, C. S. Clorose Variegada dos Citros. Bebedouro: Estação Experimental de Citricultura de Bebedouro, 1997. p.22-36.

CHANG, C.J.; GARNIER, M.; ZREIK, L.; ROSSETTI, V.; BOVÉ, J.M. Culture and serological detection of the xylem-limited bacterium causing citrus variegated chlorosis and its identification as a strain of Xylella fastidiosa. Current Microbiology, New York, v.27, p.137-142, 1993.

DAJOZ, R. Ecologia Geral.2. ed. São Paulo: Vozes, 1973. 471 p. DESCOBERTOS mais seis vetores de CVC. Revista do Fundecitrus, Araraquara, v.14, n.9, p.8-9, 1999.

HARTUNG, J.S.; BERETTA, M.J.G.; BRLANSKY, R.H.; SPISSO, J.; LEE, R.F. Citrus variegated chlorosis bacterium: axenic culture, pathogenicity, and serological relationships with other strains of Xylella fastidiosa. Phytopathology, St. Paul, v.64, p.591-597, 1994.

JACOMINO, A.P.; MODESTO, J.C.; BERETTA, M.J.; TUBELIS, A.; LEITE, R.M.V.B.C.; SALIBI, A.A. Transmissão controlada da clorose variegada dos citros (CVC). Fitopatologia Brasileira, Brasília, v.18 Suplemento, p.265, 1993.
KRÜGNER, R.; LOPES, M.T.V. de C.; SANTOS, J.S.; BERETTA, M.J.G.; LOPES, J.R.S. Transmission efficiency of Xylella fastidiosa to citrus by sharpshooters and identification of two vector species. In: CONFERENCE OF THE INTERNATIONAL ORGANIZATION OF CITRUS VIROLOGISTS, 14, Campinas. Anais... Cordeirópolis: Centro de Citricultura Sylvio Moreira, 1998, p.81.

LEE, R.F.; BERETTA, M.J.G.; HARTUNG, J.H.; HOOKER, M.E.; DERRICK, K.S. Citrus variegated chlorosis confirmation of a Xylella fastidiosa as the causal agent. Summa Phytopathologica, Jaboticabal, v.19, p.123-125, 1993.

LOPES, J.R.S.; BERETTA, M.J.G.; HARAKAVA, R.; ALMEIDA, R.P.P.; KRÜGNER, R.; GARCIA JÚNIOR, A. Confirmação da transmissão por cigarrinhas do agente causal da clorose variegada dos citros, Xylella fastidiosa. Fitopatologia Brasileira, Brasília, v.21, Suplemento, p.343, 1996.

ROBERTO, S.R.; YAMAMOTO, P.T. Flutuação populacional e controle químico de cigarrinhas em citros. Laranja, Cordeirópolis, v.19, n.2, p.269-284, 1998.

ROBERTO, S.R. Flutuação populacional de cigarrinhas (Hemiptera: Cicadellidae) vetoras de Xylella fastidiosa em pomares de laranja-doce no Estado de São Paulo. 1998. 106f. Tese (Doutorado em Produção Vegetal) - Faculdade de Ciências Agrárias e Veterinárias, Universidade Estadual Paulista, Jaboticabal, 1998.

ROBERTO, S.R.; COUTINHO, A.; LIMA, J.E.O. de; MIRANDA, V.S.; CARLOS, E.F. Transmissão de Xylella fastidiosa pelas cigarrinhas Dilobopterus costalimai, Acrogonia terminalis e Oncometopia facialis em citros. Fitopatologia Brasileira, Brasília, v.21, n.4, p.517-518, 1996.

ROBERTO, S.R.; DALLA PRIA JR., W.; YAMAMOTO, P.T.; FELIPPE, M.R.; FREITAS, E.P. de. Espécies e flutuação populacional de cigarrinhas em viveiro de citros, em Gavião Peixoto (SP). Laranja, Cordeirópolis, v.21, n.1, p.65-80, 2000.

ROSSETTI, V.; GARNIER, M.; BERETTA, M.J.G.; TEIXEIRA, A.R.R.; QUAGGIO, J.A.; BATAGLIA, O.C.; GOMES, M.P.; DE NEGRI, J.D. Resultados preliminares de estudos sobre uma nova anormalidade dos citros observada nos Estados de São Paulo e Minas Gerais. Summa Phytopathologica, Jaguariúna, v.16, p.13, 1990.

ROSSETTI, V.; GONZALEZ, M.A.; DONADIO, L.C. Histórico. In: DONADIO, L.C.; MOREIRA, C.S. (Ed.) Clorose variegada dos citros. Bebedouro: Estação Experimental de Citricultura, 1997. p.1-21.

Rev. Bras. Frutic., Jaboticabal - SP, v. 24, n. 2, p. 389-394, agosto 2002 